\title{
CDMO
}

\section{Comparação da densidade mineral óssea em mulheres praticantes de hidroginástica e sedentárias na pós-menopausa}

\section{Sandor Balsamo - CREF 00157 - DF \\ Universidade Católica de Braślia (UCB - DF) \\ Universidade Nacional de Brasilia (UnB - DF) \\ sandorbalsamo@ciaathletica.com.br}

\section{Roberto Simão - CREF 2486 - RJ}

Escola de Educação Física e Desporto Universidade Federal do Rio de Janeiro (EEFD/UFRJ). robertosimao@ig.com.br

\section{Martim Bottaro Marques - CREF 00011 - DF}

Universidade Nacional de Brasília (UnB - DF)

martimbottaro@unb.br

\section{Ana Patrícia de Paula - CRM 5791}

Coordenadora do ambulatório de osteoporose do Hospital Universitário de Brasília (HuB - DF) anapaty@unb.br

João Lindolfo Borges - CRM 2885

Universidade Católica de Brasília UCB - DF

joaolindolfo@metabolismo.com.br

BALSAMO, S.; SIMÃO, R.; MARQUES M.B; PAULA, A.P.; BORGES, J.L.; Comparação da densidade mineral óssea em mulheres praticantes de hidroginática e sedentárias na pós-menopausa. Fitness \& Performance Journal, v.5, n 4, p. 210-214, 2006.

Resum• - O objetivo deste estudo foi comparar a densidade mineral óssea (DMO) em mulheres praticantes de hidroginástica e mulheres sedentárias. Fizeram parte da amostra 48 mulheres, sendo 22 praticantes de hidroginástica $(54,5 \pm 3,3$ anos), e 26 sedentárias $(52,0 \pm 3,3)$. Todas as participantes do estudo estavam na pós-menopausa e fazendo terapia de reposição hormonal há, no mínimo, um ano. A densitometria óssea foi feita por um aparelho da marca lunar, modelo DPX-IQ. Foi obtida a DMO da coluna lombar (L2-L4), do fêmur (colo do fêmur e quadril total) e antebraço. Para a análise dos dados utilizou-se, como tratamento estatístico, a análise de variância (one-way ANOVA) para amostras independentes. Nas praticantes de hidroginástica comparadas com as sedentárias, a DMO foi mais elevada $(p<0,05)$ em L2-L4 e quadril total, enquanto nos outros sítios analisados não houve diferença significativa. Concluindo, esses resultados sugerem que a hidroginástica mostrou ser positiva para uma maior DMO em relação à população sedentária nos sítio do L2-L4 e quadril total.

Palavras-chave: Atividade aquática, Hidroginástica, Densidade mineral óssea, Menopausa.

Endereço para correspondência:

Rua Manoel Vitorino, 533 - Piedade - Prédio MR Térreo - CEP 20748-900. Rio de Janeiro

Data de Recebimento: Maio / 2006

Data de Aprovação: Junho / 2006

Copyright@ 2006 por Colégio Brasileiro de Atividade Física Saúde e Esporte. 
Comparison of the bony mineral density in practising women of water aerobics and sedentary in post-menopause

The purpose of this study was to compare the bone mineral density (BMD) between callisthenic aquatic exercises group and sedentary group. The investigated group was composed by 48 women, 22 practitioners of callisthenic aquatic training (54,5 $\pm 3,3$ years), and 26 sedentary women (52,0 $\pm 3,3$ years). All of them were in the postmenopausal period and had been using hormone therapy for over one year. The dual-energy $X$-ray absorptiometry was measured by lunar, model DPX-IQ. The BMD were measured in the spine (L2-L4), femoral neck, total hip and forearm. The data analysis was one way ANOVA to independent sample $(p<0.05)$. There was significant difference between the group of callisthenic aquatic exercises and the sedentary control group in spine (L2-L4) and total hip. We conclude that callisthenic aquatic exercises showed to be positive to BMD if compared to the sedentary group.

Keywords: Aquatic activity, Menopause, Sedentary.
La comparación de la densidad mineral ósea practicando a las mujeres de aerobic de agua y sedentario en la poste-menopausia

El propósito de este estudio era comparar el hueso la densidad mineral (BMD) entre el grupo del ejercicio acuático y el grupo sedentario. El grupo investigando estaba compuesto por 48 mujeres, 22 el grupo de entrenamiento acuático (54,5 $\pm 3,3$ años), y 26 grupo sedentario (52,0 $\pm 3,3$ años). Todos los grupos investigando estaban en el periodo del post menopausal y usan con la terapia de la hormona encima de un año. El absorptiometry de Radiografía de dualenergía era moderado por DPX-IQ lunar, ejemplar. El BMD sea moderado en la espina (L2-L4), cuello femoral, cadera total y antebrazo. El análisis de los datos era una manera ANOVA a la muestra independiente $(p<0,05)$. había diferencia significante entre el grupo del ejercicio acuático y el mando del grupo sedentario en la espina (L2-L4) y la cadera total. Nosotros concluimos ese la muestra del ejercicio acuática para ser positiva a BMD cuando comparó a las mujeres sedentarias.

Palabras-Clave: la actividad acuática, la menopausia, el hueso, la densidad mineral, sedentario.

\section{INTRODUÇÃO}

A osteoporose é um distúrbio osteo-metabólico, caracterizado pela diminuição da densidade mineral óssea (DMO), com deterioração da micro-arquitetura óssea, levando a um aumento da fragilidade esquelética e, conseqüentemente, do risco de fraturas. A osteoporose constitui-se, atualmente, em um dos maiores problemas de saúde pública dos países desenvolvidos e em desenvolvimento. Nos Estados Unidos da América (EUA), as fraturas por osteoporose chegam a 1,3 milhões ao ano, com um custo de 13,8 bilhões de dólares anuais. A probabilidade de ocorrer uma fratura por osteoporose após os 50 anos, e até o fim da vida, é de $40 \%$ nas mulheres e $13 \%$ nos homens (ACSM, 1995).

Estudos evidenciam que a atividade física é um importante fator na manutenção da massa óssea (ACSM, 1995; ANDREOLI et al., 2001; O'BRIEN, 2001; HENDERSON et al., 1998; KELLEY, 1998). A força mecânica proporcionada pelo exercício físico regular estimula a atividade dos osteoblastos, por meio do efeito piezoelétrico, ocasionando, assim, um estímulo na incorporação do cálcio no osso. Esse processo provoca um aumento das trabéculas ósseas, fazendo com que haja o fortalecimento dessas estruturas, ocorrendo assim o aumento da DMO (BALSAMO \& SIMÃO, 2005).

A atividade física no meio aquático também tem sido direcionada para mulheres idosas, principalmente aquelas com osteoporose, pois é uma atividade sem riscos de queda e, conseqüentemente, sem risco de fratura (BAUM, 2000; CAMPION, 2000). Vários autores têm citado os benefícios da hidroginástica na melhora da aptidão física (força, resistência, flexibilidade e composição corporal) e no tratamento físico de diversas doenças (BAUM, 2000; CAMPION, 2000; MAZO et al., 2001). Matsudo \& Matsudo (1991) citam que a prática da hidroginástica proporciona uma redução da freqüência cardíaca, da pressão arterial, da atividade da renina plasmática, da aldosterona e incrementa o retorno venoso. Além desses benefícios para o organismo, ela é indicada para pacientes com limitações de mobilidade (artrose, artrite), dificuldade de sustentação do peso (obesidade), reabilitação músculo-esquelética, enfermidades de coluna e osteoporose.

Estes benefícios podem ser explicados pela pressão nos tendões, ligamentos e periósteo. Segundo Matsudo \& Matsudo(1991), o estímulo da DMO ocorre na junção osteo-tendínea. Mazo et al. (2001) complementam que os exercícios que envolvem uma pressão sobre a estrutura óssea e que trabalhem contra a resistência, tais como movimentos de empurrar e puxar, podem estimular o desenvolvimento ósseo. Dessa forma, atuam, positivamente, para os pacientes com osteoporose.

A hidroginástica tem sido indicada para pacientes com osteoporose, por ser um exercício com pouco risco de queda 


\begin{tabular}{lcc}
\hline \multicolumn{1}{c}{ Variáveis } & Hidroginástica & Sedentárias \\
\hline Idade (anos) & $(\mathrm{n}=22)($ Média $\pm \mathrm{DP})$ & $(\mathrm{n}=26)$ (Média $\pm \mathrm{DP})$ \\
\hline IMC (peso/altura $\left.{ }^{2}\right)$ & $54,5 \pm 3,3^{*}$ & $52,0 \pm 3,4$ \\
\hline Tempo de menopausa (anos) & $24,7 \pm 2,7$ & $24,0 \pm 3,4$ \\
\hline TRH (anos) & $5,1 \pm 2,5$ & $3,5 \pm 2,8$ \\
\hline
\end{tabular}

Onde: ${ }^{*} p<0,05$ entre hidroginástica e sedentárias

$D P=$ Desvio padrão

IMC = índice de massa corpórea

$T R H=$ terapia de reposição hormonal

(CAMPION, 2000). Sinaki (1989) sugere que sejam feitos exercícios de reabilitação dentro da água somente em casos em que a perda da massa óssea tenha sido severa. Ressalta-se a escassez de estudos experimentais citando os reais benefícios da hidroginástica e seu estimulo osteogênico. As pesquisas sobre o meio aquático restringem-se, na sua maioria, à análise dos efeitos da natação sobre a densidade mineral óssea. Devido a esse contexto, verifica-se a necessidade de melhor conhecer a relação da hidroginástica e seus efeitos na DMO.

Portanto, o objetivo deste estudo é comparar a DMO de muIheres na pós-menopausa praticantes de hidroginástica com a DMO de mulheres na pós-menopausa sedentárias.

\section{MATERIAIS E MÉTODOS}

Este trabalho foi aprovado pelo comitê de ética da Universidade Católica de Brasília. A amostra foi composta de 48 mulheres saudáveis na pós-menopausa (47 a 61 anos). Todas as mulheres eram residentes em Brasília, e participaram do estudo de forma voluntária. Todas foram informadas do objetivo do estudo, dos procedimentos, dos possíveis desconfortos, dos riscos e de seus benefícios, antes de assinarem o termo de consentimento, conforme Resolução do Conselho Nacional de Saúde (196/96). A amostra foi dividida em dois grupos: 1) 22 mulheres participantes de hidroginástica; 2) 26 mulheres sedentárias.

Para que as mesmas participassem dos grupos do estudo foram adotados os seguintes critérios de inclusão: a) mulheres na menopausa há, no mínimo, um ano; b) mulheres em terapia de reposição hormonal (TRH) há, no mínimo, um ano; c) muIheres pós-menopausadas praticantes de hidroginástica há, no mínimo, um ano; e) mulheres pós-menopausadas sedentárias. Foram adotados os seguintes critérios de exclusão: a) doenças ou medicamentos que afetam o metabolismo ósseo, exceto suplementação de cálcio e TRH; b) mulheres com índice de massa corpórea (IMC) acima de 30.
As praticantes de hidroginástica seguiram uma metodologia de treinamento com uma freqüência de três vezes por semana, uma intensidade moderada (60 a 80\% da freqüência cardíaca máxi$\mathrm{ma}$ ). Os exercícios das aulas visavam trabalhar principalmente os grandes grupos musculares, com movimentos de empurrar e puxar, saltos e deslocamentos. As mulheres sedentárias eram pacientes do Hospital Universitário de Brasília (HUB).

As voluntárias que participaram da pesquisa foram convidadas a comparecer à unidade de densitometria óssea de Brasília e assinar o termo de consentimento. Em seguida, os testes foram aplicados na seguinte ordem: peso, estatura e DXA. Um único médico realizou a análise dos exames, sem informação sobre a atividade física das participantes. O questionário de histórico de saúde e atividade física foi aplicado no intuito de obter dados descritivos das amostras.

A densitometria óssea foi feita por um aparelho da marca Lunar, modelo DPX-IQ. Foi obtida a DMO da coluna lombar (L2-L4), fêmur (colo do fêmur e quadril total), antebraço (rádio ultradistal, rádio $33 \%$ e rádio total). Antes dos testes, os aparelhos foram calibrados conforme as recomendações do fabricante.

Essa é uma pesquisa do tipo analítica transversal e causal comparativa. Foi feito o teste de Kolmogorov Smirnof para avaliar a normalidade da amostra. Portanto, para a análise dos dados, utilizou-se, como tratamento estatístico, a ANOVA para medidas repetidas em amostras independentes. $O$ nível de significância adotado foi $p<0,05$.

\section{RESULTADOS}

Todas as participantes da pesquisa estavam na pós-menopausa e fazendo TRH há, no mínimo, um ano. Na Tabela 1 são apresentadas as variáveis com as características físicas, clínicas, médias e os desvios padrões (DP) das variáveis: idade, índice de massa corpórea (IMC), tempo de menopausa e tempo de 


\begin{tabular}{ccc}
\hline DMO $(\mathrm{g} / \mathrm{cm} 2)$ & Hidroginástica & Sedentárias \\
\hline & $\mathrm{n}=22$ (Média $\pm \mathrm{DP})$ & $\mathrm{n}=26$ (Média $\pm \mathrm{DP})$ \\
\hline L2-L4 & $1,294 \pm 0,112^{*}$ & $1,081 \pm 0,110$ \\
\hline Colo & $0,982 \pm 0,075$ & $0,927 \pm 0,081$ \\
\hline QT & $1,049 \pm 0,089^{*}$ & $0,943 \pm 0,129$ \\
\hline RUD & $0,365 \pm 0,031$ & $0,345 \pm 0,045$ \\
\hline R33\% & $0,690 \pm 0,032$ & $0,655 \pm 0,660$ \\
\hline Rtotal & $0,547 \pm 0,031$ & $0,519 \pm 0,054$ \\
\hline
\end{tabular}

Onde: ${ }^{*} p<0,05$ entre hidroginástica e sedentárias

$\mathrm{DP}=$ Desvio padrão

L2-L4 = vértebras lombares

colo $=$ colo do fêmur
QT = quadril total

RUD = rádio ultra-distal

R33\% = rádio 33\%

Rtotal $=$ rádio total
TRH. Com a análise desses dados, verifica-se que as mulheres praticantes de hidroginástica eram significativamente mais velhas que a do grupo de sedentárias, porém apresentavam resultados similares com relação ao índice de massa corpórea (IMC), tempo de menopausa e tempo de TRH.

Na Tabela 2 são apresentados os resultados da DMO do grupo de mulheres praticantes de hidroginástica e do grupo de sedentárias. Observou-se uma diferença significativa $(p<0,05)$ entre a média da DMO das praticantes de hidroginástica nos sítios de L2-L4 e do quadril total. Nos outros sítios avaliados verificou-se uma média maior nas praticantes de hidroginástica do que nas sedentárias, porém não houve diferença significativa.

\section{DISCUSSÃO}

Os principais resultados obtidos (Tabela 2) demonstraram que as praticantes de hidroginástica têm uma massa óssea significativamente maior nos sítios da coluna lombar (L2-L4) e no fêmur (quadril total). Não se obteve diferença significativa na DMO em outras regiões avaliadas, mas, mesmo assim, observou-se uma maior DMO.

Em uma das poucas pesquisas relacionando treinamento aquático com características de hidroginástica com a DMO, Tsukahara et al. (1993) avaliaram 67 mulheres na pós-menopausa, que foram acompanhadas por um período de um ano. Elas participaram de exercícios no meio aquático, pelo menos uma vez por semana. Os exercícios propostos foram saltos, caminhadas e exercícios calistênicos ou localizados leves. A sessão de treino consistia de 10 minutos de aquecimento, 20 minutos de exercícios aeróbicos, 10 minutos de natação e 5 minutos de volta à calma. $O$ grupo controle foi de 30 mulheres. Os resultados mostraram que o grupo controle perdeu $1 \%$ de DMO da coluna lombar (L2-L4), e o grupo treinado obteve um aumento de 1,55\% na DMO lombar. Os autores acrescentam que o treinamento no meio aquático é um importante fator na prevenção de perda de massa óssea.

O presente estudo demonstrou similaridades nos resultados com a pesquisa de Tsukahara et al. (1993), no sítio da coluna lombar (L2-L4). Ainda foi demonstrada no presente estudo uma diferença significativa na região do quadril total, região essa não avaliada na pesquisa citada.

Outra pesquisa envolvendo atividade física com características similares à hidroginástica foi realizada por Bravo et al. (1997), com 77 mulheres osteopênicas, com idade entre 50 e 70 anos, durante 12 meses. $O$ treinamento consistia de exercícios de saltos com movimentos e exercícios localizados, entre 15 e 20 repetições. A freqüência do treino foi de apenas uma vez por semana, com duração de 60 minutos. Os resultados demonstraram que as participantes melhoram as aptidões físicas cardiorrespiratórias, agilidade, flexibilidade, força e a resistência, reduzindo, assim, os fatores de risco de queda. Apesar disso, não houve diferença significativa após um ano de treinamento na DMO da coluna lombar (L2-L4) e no colo femoral. Bravo et al. (1997) justificam os resultados negativos da DMO em virtude do pouco impacto no trabalho de saltos na água, e também devido ao fato de as participantes, com o decorrer do treinamento, criarem mecanismos de adaptação para reduzir o impacto e, conseqüentemente, com menor força gravitacional dos pés de encontro ao fundo da piscina. Outro motivo pode ter sido em função de o treinamento ter ocorrido apenas uma 
vez por semana.

Os nossos resultados relacionados ao grupo de mulheres participantes da hidroginástica vão de encontro aos resultados da pesquisa experimental de Bravo et al. (1997), na qual não demonstraram uma diferença significativa na região do colo do fêmur de mulheres osteopênicas após um ano de treinamento de atividade física aquática similar à hidroginástica. Nessa pesquisa citada (BRAVO et al., 1997), a metodologia do treinamento constava de exercícios com saltos e exercícios localizados para diferentes partes dos membros superiores. Porém, eram realizados apenas uma vez por semana, diferentemente do presente estudo que houve uma freqüência semanal de treinamento de três vezes.

Na pesquisa de Bravo et al. (1997) foi citado que os saltos realizados na piscina produzem uma força de compressão na região da coluna lombar maior que uma caminhada no solo. Em meta-análise realizada por Kelley (1998), concluiu-se que treinamento aeróbico ajuda a manter a DMO da coluna lombar em mulheres pós-menopausadas, sendo que a maioria das pesquisas nessa meta-análise foi realizada com caminhadas. $\mathrm{Na}$ metodologia do nosso estudo constavam saltos, possivelmente influenciando positivamente nos sítios da coluna lombar (L2-L4) e no fêmur (quadril total).

Neste estudo foi avaliada, ainda, a região do rádio, sítio não avaliado por Bravo et al. (1997) e Tsukarara et al. (1993) em seus estudos com atividade física aquática similar à hidroginástica. Os resultados do nosso estudo não demonstraram diferença significativa nas médias da DMO das participantes de hidroginástica em relação ao grupo de sedentárias na região do rádio em nenhum dos sítios avaliados (RUD, R33\% e Rtotal). Portanto, este resultado sugere que a hidroginástica, talvez, proporcione pouco estímulo à região do antebraço (rádio RUD, R33\% e Rtotal).

Bravo et al. (1997) criticaram metodologicamente a pesquisa de Tsukahara et al. (1993). As principais observações dos autores foram em relação à parte estatística, na qual não foi citada, se houve, diferença significativa entre o grupo de mulheres que treinaram e o grupo controle. Apenas foi citada diferença nas médias.

A associação entre o nível de atividade e densidade mineral dos ossos que não suportam peso (como metacarpo, rádio e ulna) não tem evidenciado benefícios significativos (MATSUDO \& MATSUDO, 1991), porém, há correlação significativa com os ossos que suportam peso (MATSUDO \& MATSUDO, 1991; BALSAMO et al., 20001). A partir dos resultados envolvendo a região do antebraço, os quais não demonstraram uma diferença significativa na DMO das participantes de hidroginástica em relação às sedentárias, supõe-se que, nos programas de exercícios físicos das aulas de hidroginástica, não devem estar sendo realizados movimentos suficientes de flexão e extensão do punho e preensão manual para um estímulo osteogênico necessário nessa região.

\section{CONCLUSÃO}

As participantes de hidroginástica, na pós-menopausa, em uso de TRH, têm uma DMO maior que a de sedentárias, na pósmenopausa, em uso de TRH, nos sítios da coluna lombar (L2-L4) e quadril total. De acordo com os resultados deste estudo, sugere-se que os profissionais de saúde na área da hidroginástica intensifiquem o estímulo na região do antebraço (rádio - RUD, R33\% e Rtotal) e de colo do fêmur, pois foi verificado, nessa amostra, que essas regiões, tanto no aspecto clínico quanto no aspecto físico, precisam de maior atenção. Desse modo, são necessárias mais pesquisas, para que se possam avaliar com clareza os efeitos da hidroginástica na $\mathrm{DMO}$, pois a mesma pode também ser uma boa estratégia para a manutenção da massa óssea e possível tratamento da osteoporose. Além disso, é necessário que os profissionais de saúde estejam cientes de que estímulos intensos, específicos e freqüentes são necessários para estimular a osteogênese, e que tanto a contração muscular como o impacto estimulam o aumento da DMO.

\section{REFERÊNCIAS BIBLIOGRÁFICAS}

AMERICAN COLLEGE OF SPORTS MEDICINE. Osteoporosis and exercise. Medicine Science Sports Exercise, v.27, n.4, p.1-4, 1995.

ANDREOLI, A.; MONTELEONE, M.; LEON, M.V.; PROMENZIO, L.; TARANTINO, U.; LORENZO, A. Effects of different sports on bone density and muscular mass in highly trained athletes. Medicine Science Sports Exercise, v.33, n.4, p.507-511, 2001.

BALSAMO, S.; SIMÃO, R. Treinamento de força para osteoporose, fibromialgia, diabetes tipo 2, artrite reumatóide e envelhecimento. São Paulo: Editora Phorte, 2005.

BALSAMO, S.; PAULA, A.P.; BORGES, F.; MACHADO, A.H.; BORGES, J.L.C.; PRADO, M.; SAFONS, M.P. Avaliação da força muscular de membros inferiores em mulheres osteopênicas ou osteoporóticas. Anais do XXIV Simpósio Internacional de Ciências e Esporte, São Paulo, p. 150, 2001.

BAUM, G. Aquaeróbica. Manual de treinamento. São Paulo: Editora Manole, 2000.

BRAVO, G.; GUATIRE, P.; ROY, P. M.; PAYETTE, H.; GAULIN, P. Weight-bearing, water-based exercise program for osteopenic woman: its impact on bone, functional fitness, and wellbeing. Archives Physiological Medicine Rehabilitation, v.78, p.1375-80, 1997.

CAMPION, R.M. Hidroterapia: Princípios e prática. São Paulo: Editora Manole, 2000.

HENDERSON, K.N.; WHITE, C.P.; EISMAN, J.A. The role of exercise and fall risk reduction in reduction in the prevention of osteoporosis. Endocrinal Metababolism Clinical, v.27, n.2, p.369-387, 1998.

KELLEY, G. Aerobic Exercise and lumbar spine bone mineral density in postmenopausal women: A meta-analysis. Journal of American Geriatrics Society, v.46, n.20, p.143-152, 1998.

MAZO, G. Z.; LOPES, G.A.; BENEDETTI, B.T. Atividade Física e o Idoso: Concepção Gerontológica. Florianópolis: Editora Sulina, 2001.

MATSUDO, S.M.; MATSUDO, V.K.R. Osteoporose e atividade física. Revista Brasileira de Ciência e Movimento, v.5, n.3, p.33-60, 1991.

O'BRIEN, M. Exercise and osteoporosis. Journal Medicine Science, v.170, n.1, p.58-62, 2001.

SINAKI, M. Exercise and osteoporosis. Archives of Physical Medicine and Rehabilitation, v.70, n.220-229, 1989.

TSUKARARA, N.; TODA, A.; GOTO J.; EZAWA, I. Cross-section and longitudinal studies on the effect of water, exercise in controlling bone loss in Japanese postmenopausal woman. Journal Nutrition Science Vitaminol, v.40, p.37-47, 1993. 